\title{
Interaction of Energetic Particles with Large and Small Scale Instabilities
}

\author{
S. Günter ${ }^{1}$, G. Conway ${ }^{1}$, S. daGraça ${ }^{2}$, H.-U. Fahrbach ${ }^{1}$, C. Forest ${ }^{3}$, M. Garcia Muñoz ${ }^{1}$, T.

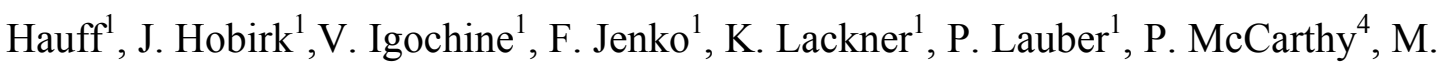 \\ Maraschek $^{1}$, P. Martin ${ }^{5}$, E. Poli ${ }^{1}$, K. Sassenberg ${ }^{4}$, E. Strumberger ${ }^{1}$, G. Tardini ${ }^{1}$, E. Wolfrum ${ }^{1}$, \\ H. Zohm ${ }^{1}$, ASDEX Upgrade Team \\ ${ }^{1}$ Max-Planck Institut für Plasmaphysik, Garching, Germany, EURATOM Association \\ ${ }^{2}$ Centro de Fusão Nuclear, Associa cão EURATOM/IST, Instituto Superior Técnico, Lisboa, \\ Portugal \\ ${ }^{3}$ Dept. of Physics, University of Wisconsin, Madison, Wisconsin, USA \\ ${ }^{4}$ Physics Department, University College Cork, Cork, Ireland \\ ${ }^{5}$ Consorzio RFX, Assozione EURATOM-ENEA per la fusione, Padova, Italy
}

e-mail contact of main author: Sibylle.Guenter@ipp.mpg.de

Abstract: Beyond a certain heating power, measured and predicted distributions of NBI driven currents deviate from each other even in the absence of MHD instabilities. The most reasonable explanation is a redistribution of fast NBI ions on a time scale smaller than the current redistribution time. The hypothesis of a redistribution of fast ions by background turbulence is discussed. Direct numerical simulation of fast test particles in a given field of electrostatic turbulence indicates that for reasonable parameters fast and thermal particle diffusion can indeed be similar. - High quality plasma edge density profiles on ASDEX Upgrade and the recent extension of the reflectometry system allow for a direct comparison of observed TAE eigenfunctions with theoretical ones as obtained with the linear, gyrokinetic, global stability code LIGKA. These comparisons support the hypothesis of TAE-frequency crossing the continuum at the plasma edge in ASDEX Upgrade H-mode discharges. - A new fast ion loss detector with $1 \mathrm{MHz}$ time resolution allows frequency and phase resolved correlation between the observed losses and low frequency magnetic perturbations such as TAE modes and rotating magnetic islands. Whereas losses caused by TAE modes are known to be due to resonances in velocity space, by modelling of the particle drift orbits we were able to explain losses caused by magnetic islands as due to island formation and stochasticity in the drift orbits.

\section{Introduction}

It has long been recognized that the interaction of fast particles with large scale magnetic perturbations can have a major influence on heating efficiencies and wall loading.

Recently, discrepancies have also been found between the predicted and observed current profile modifications due to off-axis, correlated with the increase of heating power and of 
turbulent energy losses [1-4]. It has therefore been surmised that also small scale turbulence, driven by gradients in the thermal plasma profiles, might have an effect on suprathermal particles.

ASDEX Upgrade is well equipped for studies of fast particle physics, due to its powerful and flexible heating system (up to $20 \mathrm{MW}$ NBI at $60 / 93 \mathrm{keV}$ energy; up to $6 \mathrm{MW}$ ion cyclotron resonance heating (ICRH), up to $2 \mathrm{MW}$ electron cyclotron resonance heating (ECRH)) and its well developed diagnostics. Recent relevant diagnostic upgrades consist in a high-time resolution fast ion loss detector [5], and upgrades to the reflectometry system. In parallel we have been developing the theoretical tools for the study of instabilities driven by fast particles (in the form of a linear global gyrokinetic stability code, which includes a full description of the fast particle orbits) and of the effect of background plasma turbulence on their dynamics.

\section{Current Profile Modification by off-axis NBI on ASDEX Upgrade}

To achieve steady state scenarios with reversed magnetic shear, means for current profile control are required. For ITER, off-axis current drive by negative ion based NBI (N-NBI) is been envisaged. Beam drive of current has been predicted by theory (see e.g. [6-8] and observed in many experiments (e.g., in TFTR [9,10], DIII-D [11,12], JET [13,14], JT-60U [15-17], for an overview see [18]). In most of these experiments the beam driven current was located on-axis, including those with N-NBI [16,17]. The only off-axis current drive experiments (with 2 MW NBI power) - apart from ASDEX Upgrade - have been reported by JT-60U $[15,19]$.

ASDEX Upgrade is equipped with a flexible NBI heating system, allowing on- and off-axis heating and current drive. Typical NBI current-redistribution experiments pass through a sequence: on-axis/off-axis/on-axis beam injection, with the length of the phases chosen to allow approach to resistive equilibrium (Fig. 1). The off-axis injection is accompanied by central ECRH, adjusted in power to keep the electron temperature, and hence the resistivity, approximately constant in time (Fig. 2). All experiments reported in the following were in a type-I ELMy H-mode regime and with deuterium beam injection. The two off-axis beamsources, with a beam voltage of $93 \mathrm{keV}$ and a total power of up to $5 \mathrm{MW}$ have a tangency major radius of $1.29 \mathrm{~m}$ (nominal major radius of ASDEX Upgrade: $1.65 \mathrm{~m}$ ) and are inclined vertically to be tangent, in a poloidal plane, to the half-minor radius flux surface. The on-axis 
beam sources (total power up to $15 \mathrm{MW}$ ) operate at $60 \mathrm{keV}$ (4 sources) and 93keV (2 sources) respectively. The motional Stark effect (MSE) system, the main diagnostic system for the current profile measurements, is linked to the operation of one of the more radially directed $60 \mathrm{keV}$ sources (tangency radius 0.93m) [20]. Fig. 3 shows profiles for the NBI driven current density during the on-axis and off-axis injection phases of \#18383, as predicted by the TRANSP code [21]. Although the current drive efficiency of course depends on the plasma parameters, these profiles can be considered typical for ASDEX Upgrade current drive discharges.

At sufficiently low total heating power the measured time traces of the MSE signal correspond very well to the TRANSP predictions (Fig. 4). At high triangularity $(\delta \sim 0.4)$, this regime extends up to our full off-axis power capability. As the MSE system is linked to an onaxis beam, only the signal during phases of its operation can be directly compared with the modelling results. It is evident, however, that for the case shown, the experimental MSE traces correspond - over the whole radius range - far better to signals reconstructed from TRANSP-runs including the NBI current drive with full nominal efficiency than to those neglecting it. Fig. 5 shows the modelled NBI and total current densities for two time instances, at the end of the off-axis and the second on-axis phases. Such changes in the current density profile for low power discharges are also confirmed by the observed shift in the resonance location of occasionally appearing MHD activity. In the discharge considered in Fig. 4, a small $(3,2)$ NTM has been observed. This mode is not responsible for the changes in the current profile as in several similar discharges no MHD activity was observed at all. During the off-axis beam phase, the mode rational surface is shifted inwards as observed by radial well resolved electron cyclotron emission (ECE) measurements by about $1 \mathrm{~cm}$. In discharges with ECE measurements up to the plasma centre also a shift of the location of $(1,1)$ activity was, see Fig. 6. (In these discharges the adjustment of the electron temperature was achieved by central IRCH heating during the off-axis beam phase).

In the high heating power regime (which for low triangularity $(\delta \sim 0.2)$ is reached with less than $5 \mathrm{MW}$ ) the current profile changes measured by the MSE system fall significantly short of the predictions of the TRANSP code (Fig. 7). The agreement can be substantially improved by introducing an artificial diffusion of the slowing down fast particles, with an assumed diffusivity $\mathrm{D}_{\text {fast }} \approx 0.5 \mathrm{~m}^{2} / \mathrm{s}$. Fig. 8 shows the TRANSP current density profiles at the end of the 
off-axis and on-axis injection phases, computed both with and without fast particle diffusion. Whereas for the on-axis NBI case, fast particle diffusion does not lead to a significant change of the current density profile, in case of off-axis NBI a stronger peaking of the current density profile is found. This peaking results from a significant NBI current contribution in the plasma centre, introduced by the fast particle diffusion. The additional central current is larger than to be expected from a symmetric broadening of the NBI current profile as the radial profile of the electron temperature and the corresponding beam ion slowing down time strongly influence the current driven by the beam ions (Fig. 9). In the discharge considered here, no MHD activity with the exception of type I ELMs has been observed. The MHD activity associated with the $\mathrm{q}=1$ surface has been avoided by operating at low plasma current $\left(\mathrm{I}_{\mathrm{p}}=600 \mathrm{kA}\right)$ resulting in a high $\mathrm{q}$ value at the plasma edge $\left(\mathrm{q}_{95}=6.2\right)$.

The predicted total driven current, as monitored by the loop voltage, agrees quite well with TRANSP simulations throughout all power regimes, even without the assumption of additional fast particle diffusion. The inclusion of fast particle diffusion does not significantly change this result (Fig. 10). This was to be expected as fast particle diffusion should affect the net-current drive efficiency only through higher order effects (e.g. by displacing particles into regions with different slowing-down rate).

More direct information on the radial distribution of the NBI driven currents and their temporal behaviour can be derived from the radial profiles of the parallel electric field. The latter follow from equilibrium reconstructions, using time dependent measurements of MSE angles and loop voltage. For the loop voltage profiles shown in Fig. 11, the procedure described in $[22,23]$ has been applied, using the equilibrium reconstruction procedures in the CLISTE code [24,25]. Immediately after switching from on-axis to off-axis beams (and back), the profiles show the expected behaviour: the plasma responds to the additional (reduced) current drive by a loop voltage decrease (increase) at the radial location of the respective beam deposition. Within a time of about $100 \mathrm{~ms}$, however, the loop voltage flattens again. This time is much shorter than that needed for a corresponding current redistribution (1.5 $\mathrm{s}$ according to TRANSP simulations). As no strong MHD activity is present in this discharge (except for small fishbones in the very plasma centre), current redistribution cannot have been completed within this time. On this time scale only the source of the current, the fast particles, can be redistributed. 
Obvious candidate mechanisms for a fast-particle re-distribution are Alfvén-type waves or other MHD activity. To rule out $\mathrm{m} / \mathrm{n}=1 / 1$ type MHD activity of fishbone or sawtooth type, we have carried out some of the NBI current re-distribution experiments at sufficiently high $\mathrm{q}_{95}$ values to avoid (1/1) activity. We did not find however, any significant influence of the $\mathrm{q}_{95}$ value on the observed and modelled NBI current distribution. To rule out a possible role of Alfvén waves, we have also carried out experiments varying the resonance conditions for such waves, e.g. by reducing the beam velocity. In most of our experiments we used the full beam energy of $93 \mathrm{keV}$, corresponding to a velocity parallel to the magnetic field of $2.0510^{6}$ $\mathrm{m} / \mathrm{s}$. The Alfvén velocity for the discharges considered in Figs. 7 and 11 is about $5.510^{6} \mathrm{~m} / \mathrm{s}$ in the plasma centre and $6.510^{6} \mathrm{~m} / \mathrm{s}$ at half the plasma minor radius. In the discharge discussed in Fig. 11 the beam velocity has been reduced to $69 \mathrm{keV}$, corresponding to $\mathrm{v}_{\|}=1.7$ $10^{6} \mathrm{~m} / \mathrm{s}$, below $\mathrm{v}_{\mathrm{A}} / 3$. The fast particle pressure is about $25 \%$ of the total plasma pressure $\left(\beta_{\mathrm{pol}}\right.$ $=1$ ) in this discharge. In none of the discharges we found any evidence for Alfvén wave activity, in spite of a dedicated search with Mirnov, SXR and reflectometry diagnostics. It should also be noted that observations of Alfvén activity for $\mathrm{v}_{\|}<\mathrm{v}_{\mathrm{A}} / 3$ as reported from other experiments appear to be restricted so far to hollow-q profiles (see e.g. [26]), a situation not pertinent to our experiments.

The results described above indicate a redistribution of fast ions, driven by turbulent fluctuations correlated with the thermal transport. Such a redistribution would fill in the fast particle distribution in the centre, but would also bring energetic ions into the outer, cooler regions, where they would undergo a faster slowing-down, resulting in an overall reduced current drive efficiency. It should be noted that this spreading would have a much smaller effect on other measures of the fast ion population, like their contribution to plasma heating or the neutron production. The latter effects weight more strongly the highest velocity phase of the slowing down history. To affect these quantities would therefore require a much faster redistribution of the beam ions than needed to influence the profile of the NBI driven current. Our experiments do not contradict earlier experiments reporting classical fast particle slowing behaviour either, as all these experiments were done at very low total heating power, see, e.g., [18] and references cited therein.

\section{Turbulent ExB advection of fast test particles}


The apparent correlation of the fast ion redistribution with the thermal heat transport described above has raised interest in the more general question of the response of suprathermal particles to background plasma turbulence. It has been argued [27-29] that the interaction of fast ions with the background turbulence should be small - mainly because of their large gyroradius which gyro-averages out electrostatic potential fluctuations. However, the recent de-correlation trajectory theory put forward by M. Vlad et al. [30] has shown this picture to be oversimplified, as gyro-averaging also leads to a broadening of the zone of influence of each individual eddy. For a more direct assessment we have carried out direct numerical simulation of an assembly of test particles in a constant background magnetic field and a prescribed turbulent spectrum of electrostatic fluctuations, using either a full-orbit, or a gyrokinetically averaged model of particle motion (with no significant difference in the results between the two models). The problem is defined by two characteristic parameters: the Kubo number, suitably defined for this case by $K=V_{E \times B} \tau_{c} / \lambda_{c}\left(V_{E \times B}:\right.$ ExB velocity, $\tau_{c}, \lambda_{c}$ : correlation time and length of the turbulent potential), and $\rho / \lambda_{c}$ ( $\rho$ being the gyroradius of the test particles). Core turbulence is typically found to peak around $\pi / k_{y} \rho_{s} \approx 10-15$, corresponding to $\rho / \lambda_{c} \approx 2$ for thermonuclear $\alpha$-particles and correspondingly smaller values for our beam ions. The results of our test particle simulations, illustrated in Fig. 12, show a very large reduction of turbulent diffusion by finite gyro-radius effects for relevant $\rho / \lambda_{c}$ - values only at small Kubo numbers. Based on simulations with the nonlinear gyrokinetic code GENE we expect the Kubo numbers under realistic experimental conditions to be of the order of unity or even larger. In this range the diffusion coefficient for fast particles is not significantly different from that of cold testparticles. That way, one finds that for Kubo numbers up to about unity, the test particle transport drops significantly with increasing gyroradius, but for the larger Kubo numbers typical for strong plasma turbulence, the dependence on the gyroradius is relatively weak (as long as the gyroradius is smaller than a few perpendicular correlation lengths of the turbulence). These numerical results can be also understood in terms of a modified form of decorrelation trajectory theory [31]. Regarding our experimental findings, these results support our interpretation in terms of a correlation between increased thermal transport and enhanced fast particle diffusion leading to a broadening of the distribution of NBI driven currents.

A direct comparison with the experimental results would require a more complete physics description, in particular the inclusion of the dynamics parallel to the magnetic field lines. For 
these investigations we have included the fast ions as a separate species into the gyrokinetic turbulence code GENE. Describing the fast ions as tracer particles that are redistributed by a turbulent background plasma, first numerical results indicate again a significant redistribution of fast ions [32].

\section{Damping of TAE modes}

The nature and magnitude of the dominating TAE damping mechanism are decisive for the amplitude of fast-particle excited TAE modes and hence for the associated particle and energy losses. Direct measurements of damping rates of externally excited TAE modes on JET showed large values, explained at that time by a gyrokinetic model as being due to radiative damping in the plasma centre [33]. Other gyrokinetic codes, developed in the meantime, and analytic models, however did not confirm this explanation [34]. An alternative explanation presupposes the intersection of the TAE frequency and the continuum Alfvén spectrum at the plasma edge. This possibility depends sensitively on edge profiles and could not be ruled out within the experimental uncertainties of the density measurements. To examine quantitatively this and other aspects of the damping and excitation of Alfvén waves we have developed a linear, fully gyrokinetic, non-perturbative eigenvalue code, including realistic fast particle orbits: LIGKA [35]. It has been extensively benchmarked against other codes in their respective range of validity. With this code, indeed, satisfactory quantitative agreement with the measured damping rates could be achieved under assumptions leading to closed gaps in the Alfvén spectrum.

Conclusive experiment-theory comparisons require, however, accurate measurements of both the density profiles and the eigenfunction of the excited modes. Density profiles are available with high quality, in particular at the plasma edge, on ASDEX Upgrade using a combination of Thomson scattering, Lithium beam and reflectometry data. A density profile for \#21007 (4.23 s) is given in Fig. 13. Of particular interest for the stability of TAE modes is the plasma edge, where highly resolved Li beam spectroscopy measurements are available. Fig. 14 shows the corresponding Alfvén spectrum for $n=4$ modes as resulting from an equilibrium reconstruction and the density profile of Fig. 13. This spectrum indicates that the TAE gap becomes closed at the plasma edge. This result does not change if the density profile is varied within the experimental error bars. 
Eigenfunction measurements in the core with SXR can now be complemented by reflectometry measurements in the edge region. In a frequency hopping mode - under conditions of stationary TAE activity - reflectometry measurements probe the density fluctuations at different radial locations, using correlation techniques to reconstruct the envelope of the TAE eigenfunction [36,37]. The experimental eigenfunction shown in Fig. 15 gives the cross phase between magnetic and reflectometry signals for an $n=4$ TAE perturbation excited by ICRH heating in a low density discharge, where the peaked density profile allows for reflectometry measurements up to the plasma core (for details see [38]). The theoretical eigenfunction shown corresponds to the density perturbation (according to Eq. 1 of [39]) caused by the least damped $n=4$ mode as derived by LIGKA. The two eigenfunctions are in reasonable agreement; note in particular the increase in the mode amplitude close to the plasma edge which (at least in the calculation) results from the coupling to the Alfven continuum. The observed frequency of the $n=4$ TAE mode is $230 \mathrm{kHz}$. As strong TAE activity is usually found in low density purely - ICRH heated discharges, we do not have available plasma rotation measurements which would require NBI heating. We therefore estimate the Doppler shift from the frequency difference between the $n=4$ and $n=5$ TAE mode which is found to be $12 \mathrm{kHz}$. As the frequency of the least damped $\mathrm{n}=4$ TAE mode from LIGKA is $185 \mathrm{kHz}$, inclusion of the Doppler shift would result in $227 \mathrm{kHz}$.

In fact, the LIGKA results suggest that only for closed gaps TAE damping rates for the least damped modes can be as large as observed in the JET antenna experiments discussed above.

\section{Interaction of fast particles with magnetic perturbations}

New insights into the interaction between fast particles and large scale magnetic perturbations have been gained by the deployment of a fast ion loss detector [5] following the principle design used on TFTR [40] and later W7-AS [41]. In addition to the observation with a CCD camera an array of photomultipliers, with a bandwidth of $1 \mathrm{MHz}$ is used. The high time resolution of the photomultipliers allows not only frequency but also phase correlation of particle losses with MHD fluctuations, and such correlations have in fact been clearly observed not only for TAEs, but also for lower frequency magnetic perturbations (NTMs, double tearing modes, ELMs). In case of NBI produced particles the capability of intermittent operation of the sources, with a - ramp-down time of less than $50 \mu \mathrm{s}$, gives additional information on the origin of the observed fast particles. MHD perturbations leading to large islands give origin to losses to the detector, which are modulated with the frequency and 
phase locked with the mode. In these cases the phase velocity of the mode is much smaller than the fast particle velocity. Fig. 16 shows a CCD camera picture of the detector during a double-tearing event, for standard, co-directed beam orientation. The luminous patches can be assigned to fixed pitch-angle and energy windows. The three most prominent ones correspond (from the left) to high field side deposition of the more radial $93 \mathrm{keV}$ source (source \#8), low field side deposited particles of the more tangential source (source \#7) and low field side deposition of \#8. All three show a strong modulation with the amplitude closely related to that of the magnetic perturbation [42].

Intermittent operation of source \#8 shows an immediate (within 5-15 $\mu$ s) complete decay of the signal, demonstrating that particles are lost within the first 2-3 toroidal orbits (Fig. 17 left). To explain these observations, simulations with the GOURDON code [43] have been performed to follow the drift orbits of the NBI ions in realistic ASDEX Upgrade geometry. The NBI deposition profile has been taken from FAFNER [44] simulations with the ASDEX Upgrade beam geometry. According to these simulations, the rapid losses result from particles deposited on the high field side well inside the separatrix. Due to the torus drift displacement of their orbits however, they can cross the separatrix readily on the low field side. The modulation with the island frequency can be explained by the formation of drift islands with $(3,1)$ and $(4,1)$ helicities. Co-directed particles deposited inside the separatrix on the low field side, on the other hand, can reach the detector only after being displaced to outer flux surfaces. In fact, low field side deposited particles of source \#8 respond with a much larger delay (of the order of $5 \mathrm{~ms}$ ) to the switching-off of the source (Fig. 17, right). From the NBI deposition profile and the measured pitch angle (pitch angle scattering is not important in the corresponding energy and temperature range), the radial range over which the observed ions are deposited in the plasma can be estimated to extend to about $10 \mathrm{~cm}$ inside the separatrix. According to drift orbit calculations using the Orbit [45] and the Gourdon code, the observed temporal decay can be explained to be due to a region of orbit stochasticity [46], leading to drift orbit losses proceeding over many (order $>10^{2}$ ) toroidal particle transits, see Fig. 18. The decay time is probably limited by the collisional slowing down of the fast particle population (which, in the edge region, would also correspond to a few ms). The observation of a strong increase of fast ion losses caused by magnetic islands reported here is consistent with the observation of increased heat loads to the limiters found usually on ASDEX Upgrade as soon as large magnetic islands appear. 
Frequency and phase related modulation of losses were also observed for more energetic, ICRH produced particles, in a pitch-angle range corresponding to strong trapping. Calculations with the HAGIS code [47] yield a possible explanation in terms of a resonance of the bouncing particle orbits with a (3,2) NTM (Fig. 19). For particles of certain pitch angle and energy (within the experimentally expected range) bounce orbits return nearly to the same poloidal and toroidal location. These particles therefore find a fixed phase of the magnetic perturbation after an integer number $n$ of bounces (assuming the magnetic perturbation for the moment as stationary). This implies that the (radial) motion induced by the perturbed field lines, which usually averages to zero over a few bounce times, can lead to a net radial drift of the trapped-particle orbits, inwards or outwards depending on the phase of the orbit with respect to the island [48]. This resonance condition can be satisfied also in the presence of a

non-vanishing $v_{\text {prec }}-2 \pi R /\left(n \tau_{\text {bounce }}\right)$, which has to be equal to the toroidal phase velocity of the mode.

\section{References}

[1] J. Hobirk et al., Off-axis neutral beam current drive experiments on ASDEX Upgrade and JT-60U, $30^{\text {th }}$ EPS conference, St. Petersburg, Russia, 2003, O-4.1B

[2] S. Günter et al., Conditions for NBI current profile control on ASDEX Upgrade, $31^{\text {st }}$ EPS conference, London, UK, 2004, O1.02

[3] S. Günter et al., Current profile modification by off-axis NBI on ASDEX Upgrade, $32^{\text {st }}$ EPS conference, Tarragona, Spain, 2005, P-4.075

[4] S. Günter et al., Nucl. Fusion 45, S98 (2005)

[5] M. Garcia Muñoz, PhD thesis, Fast response scintillator based detector for MHD induced energetic ion losses in ASDEX Upgrade, Ludwig-Maximilian-Universität of Munich (2006)

[6] T. Ohkawa, Nucl. Fusion 10185 (1970)

[7] J.G. Cordey et al., Nucl. Fusion 19249 (1979)

[8] D.R. Mikkelsen et al., Nucl. Technology/Fusion 4237 (1983)

[9] M.C. Zarnstorff et al., Phys. Rev. Lett. 60, 1306 (1988)

[10] T.C. Simonen et al., Phys. Rev. Lett. 61, 1720 (1988)

[11] R.J. Hawryluk, Plasma Phys. Control. Fusion 331509 (1991)

[12] C.B. Forest et al., Phys. Rev. Lett. 73, 2444 (1994) 
[13] J.G. Cordey et al., Plasma Phys. Contr. Fusion 30, 1625 (1988)

[14] C.D. Challis et al., Nucl. Fusion 29563 (1989)

[15] S. Ide et al., Non-inductive current drive experiments for profile control in JT-60U, in Plasma Physics and Controlled Nuclear Fusion Research 1994 (Proc. 15 ${ }^{\text {th }}$ Int. Conf. Seville, 1994), Vol. 1, p. 641, IAEA Vienna (1995)

[16] T. Oikawa et al., Nucl. Fusion 40435 (2000)

[17] T. Oikawa et al., Nucl. Fusion 411575 (2001)

[18] W. Heidbrink and G.J. Sadler, Nucl. Fusion 34535 (1994)

[19] T. Suzuki et al., Off-axis current drive and current profile control in JT-60U, in Fusion Energy 2006 (Proc. 21 ${ }^{\text {st }}$ Int. Conf. Chengdu, 2006) (Vienna: IAEA) CD-Rom file EX/6-4 and http://www-naweb.iaea.org/napc/physics/FEC/FEC2006/html/index.html

[20] R.C. Wolf et al., Motional Stark Effect Polarimetry for the Determination of the ASDEX Upgrade Current Density Profile, Europhysics Conference Abstracts (Proc. 24th Eur. Conf.

Berchtesgaden 1997), p1509-1512, June 1997

[21] A. Pankin et al., Comput. Phys. Commun. 159 , 157 (2004)

[22] C.B. Forest et al., Phys. Rev. Lett 73, 2444 (1994)

[23] J.K. Anderson et al., Phys. Plasmas 11, L9 (2004)

[24] P.J. McCarthy et al., Non-inductive current drive analysis on ASDEX Upgrade using the CLISTE equilibrium code with loop voltage profile reconstruction, $33^{\text {st }}$ EPS conference, Rome, Italy, 2006, P2.144

[25] P.J. McCarthy, Phys. Plasmas 6, 2834 (1999)

[26] R. Nazikian et al., Energetic particle driven modes in advanced tokamak regimes on JET, in Fusion Energy 2004 (Proc. $20^{\text {th }}$ Int. Conf. Vilamoura, 2004) (Vienna: IAEA) CD-Rom file EX/5-1 and http://www-naweb.iaea.org/napc/physics/fec/fec2004/datasets/index.html

[27] G. Manfredi and. R.O. Dendy, Phys. Plasmas 4, 628 (1997)

[28] H. Naitou, T. Kamimura and J.M. Dawson, JPS of Japan 46, 258 (1979)

[29] S.V. Annibaldi, G. Manfredi and. R.O. Dendy, Phys. Plasmas 9, 791 (2002)

[30] M. Vlad and F. Spineanu, Plasma Phys. Control. Fusion 47, 281 (2005)

[31] T. Hauff and F. Jenko, Phys. Plasmas 13, 102309 (2006)

[32] T. Dannert et al., in preparation

[33] A. Fasoli, A. Jaun, and D. Testa, Phys. Lett. A 265, 288 (2000)

[34] G. Y. Fu, H.L. Berk, and A. Pletzer, Phys. Plasmas 12, 082505 (2005)

[35] Ph. Lauber, S. Günter, S. Pinches, Phys. Plasmas 12, 122501 (2005)

[36] L. Cupido et al., Rev. Sci. Instrum. 75, 3865 (2004) 
[37] S. Da Graca et al., Localisation of MHD and fast particle modes in ASDEX Upgrade using reflectometry, 33th EPS conference, Rome, 2006, P2.146

[38] S. Da Graca et al., Localization of MHD and fast particle modes using reflectometry in ASDEX Upgrade, submitted to Plasma Phys. Control. Fusion (2007)

[39] R. Nazikian et al., Phys. Rev. Lett. 91, 125003 (2003)

[40] D. Darrow, Rev. Sci. Instrum. 66, 476 (1995)

[41] A. Werner, A. Weller, Rev. Sci. Instrum. 72, 780 (2001)

[42] M. Garcia Muñoz et al., NTM induced fast ion losses on ASDEX Upgrade, to be published in Nucl. Fusion (2007)

[43] C. Gourdon, „Programme Optimise de Calculs Numeriques Dans le Configurations Magnetique Toroidales“, CEN Fontenay aux Roses, 1970

[44] G.Lister, Techn. Rep., Max-Planck-Institut für PLasmaphysik (1985)

[45] R. White, Phys. Fluids 27, 2455 (1984)

[46] H.E. Mynick, Phys. Fluids B5, 1471 (1993)

[47] S. D. Pinches et al., Comp. Phys. Comm. 111, 131 (1998)

[48] E. Poli, in preparation 


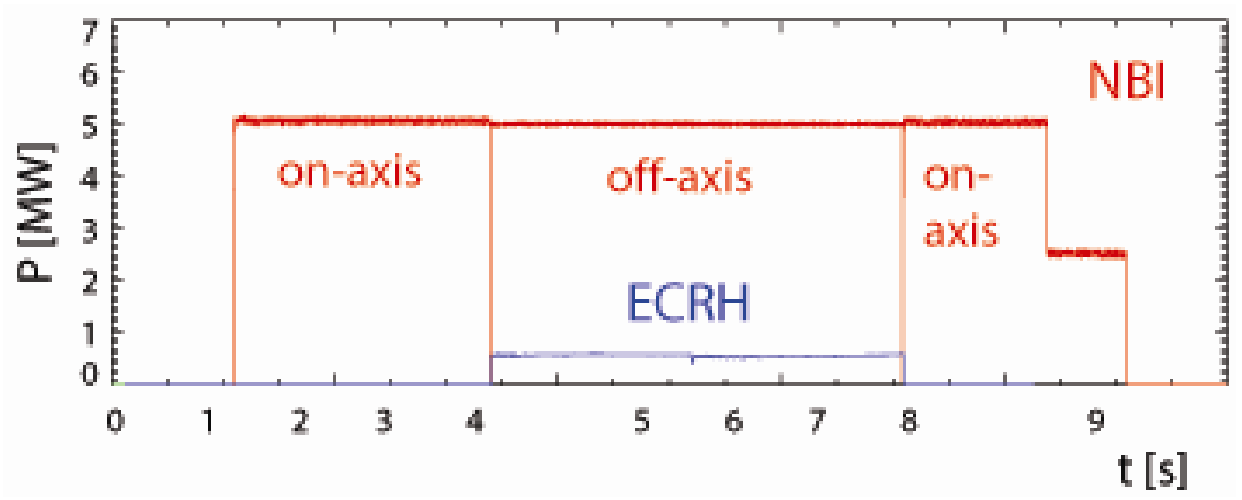

Fig. 1. Time traces for heating power in a typical NBI current redistribution discharge. The off-axis NBI is complemented by ECRH to keep the electron temperature approximately constant in time.

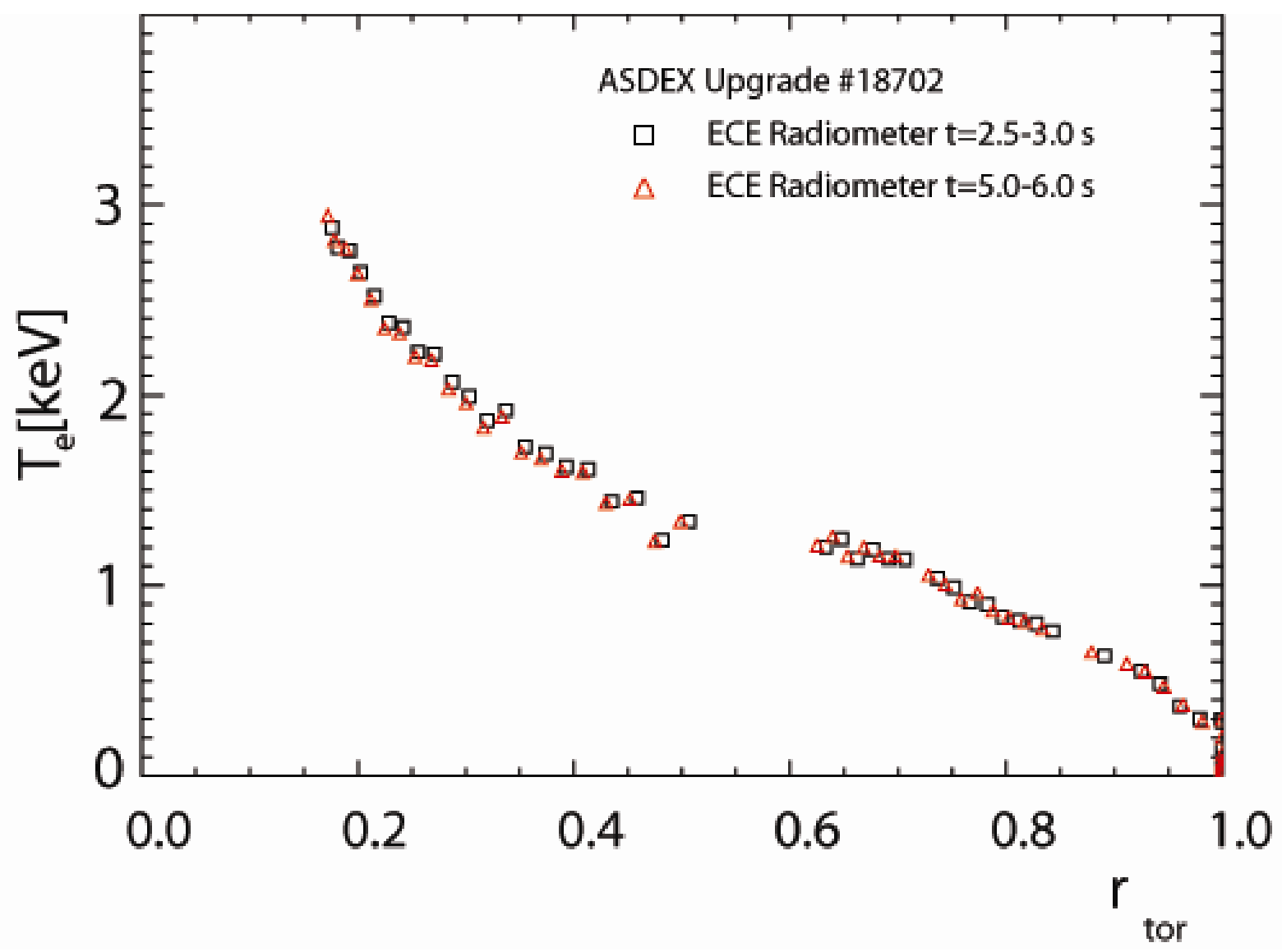

Fig. 2. Electron temperature profiles for on- and off-axis beam deposition in the discharge of Fig. 1. 


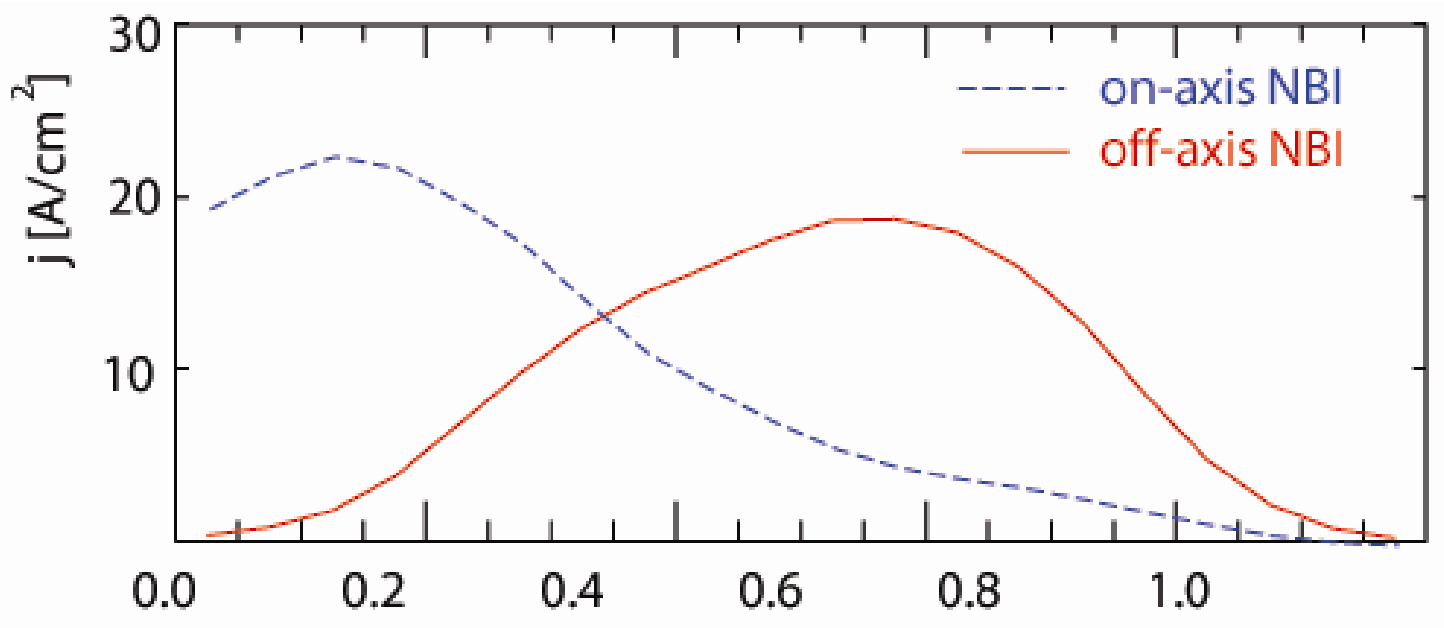

$r$ tor

Fig. 3: Typical profiles for the NBI driven current density during on-axis (dashed) and off-axis injection phases (solid), as predicted by TRANSP. This particular analysis has been performed for the ASDEX Upgrade discharge \#18383. The general shape of the current density profiles is, however, not strongly affected by the plasma parameters.

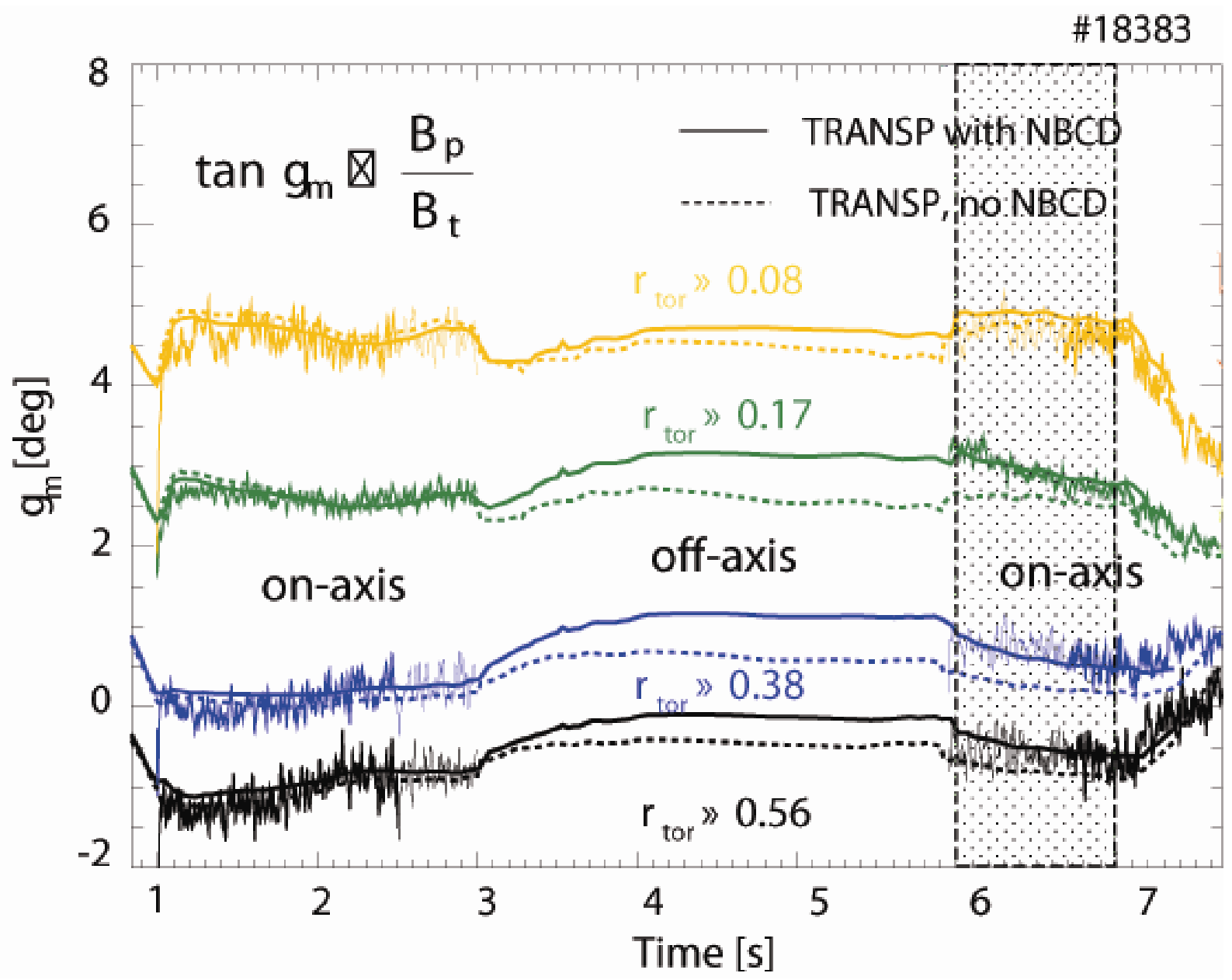


Fig. 4: Measured time traces of the MSE diagnostic at different radii compared to TRANSP predictions including (solid line) and excluding (dashed) NBI current drive. Discharge parameters: $I_{p}=800 \mathrm{kA}, B_{\mathrm{t}}=2.5 \mathrm{~T}, \delta=0.4, P_{N B I}=5 \mathrm{MW}$. Note that the current profile relaxation right after the offaxis NBI phase (highlighted) is in good agreement with corresponding TRANSP results if NBI current drive is included. (The relative error of the MSE angles is about 0.2 degrees.)

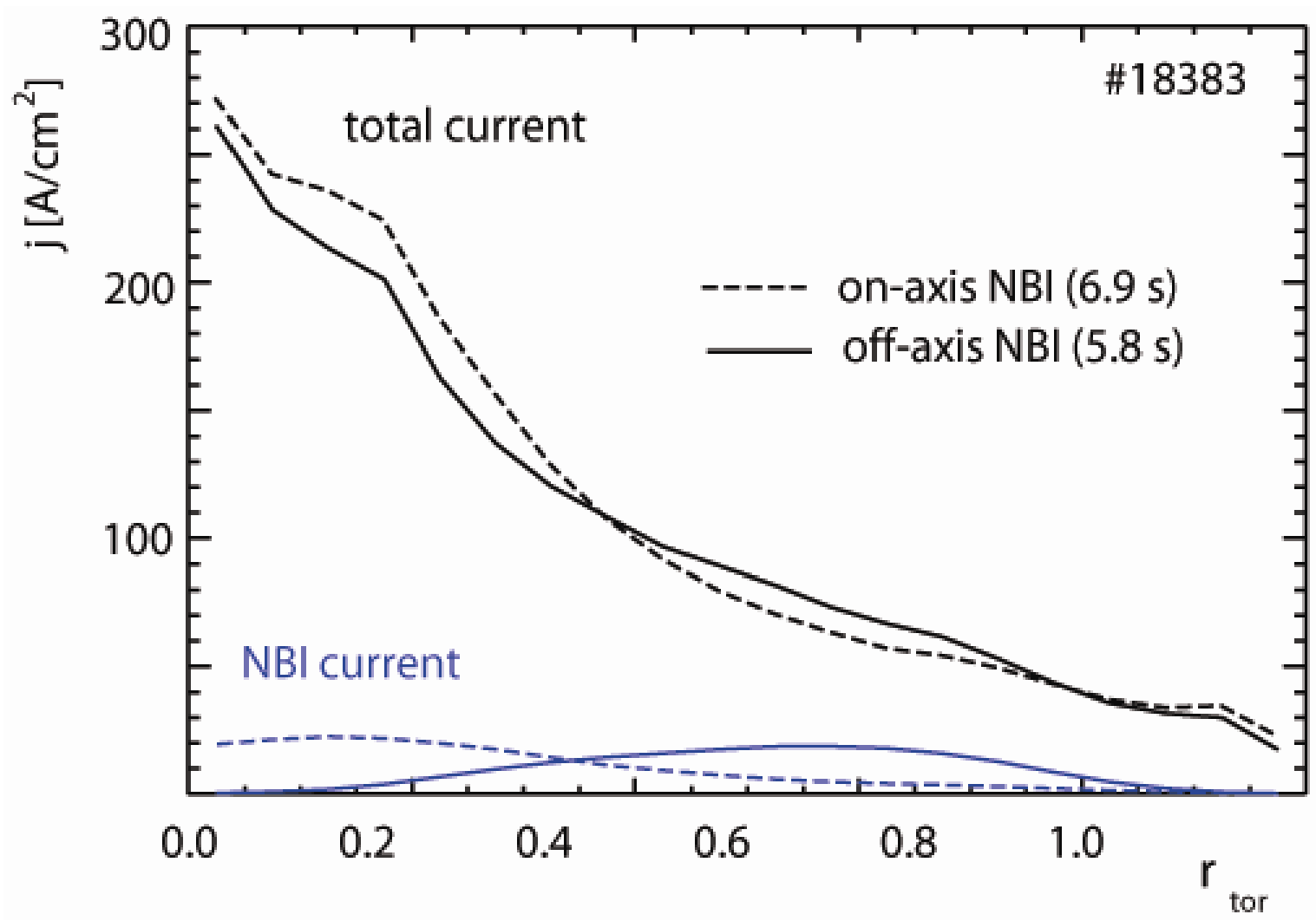

Fig. 5: TRANSP results for NBI and total current density profiles at the end of the off-axis (solid) and the on-axis phase (dashed) for the same discharge as in Fig. 4. 


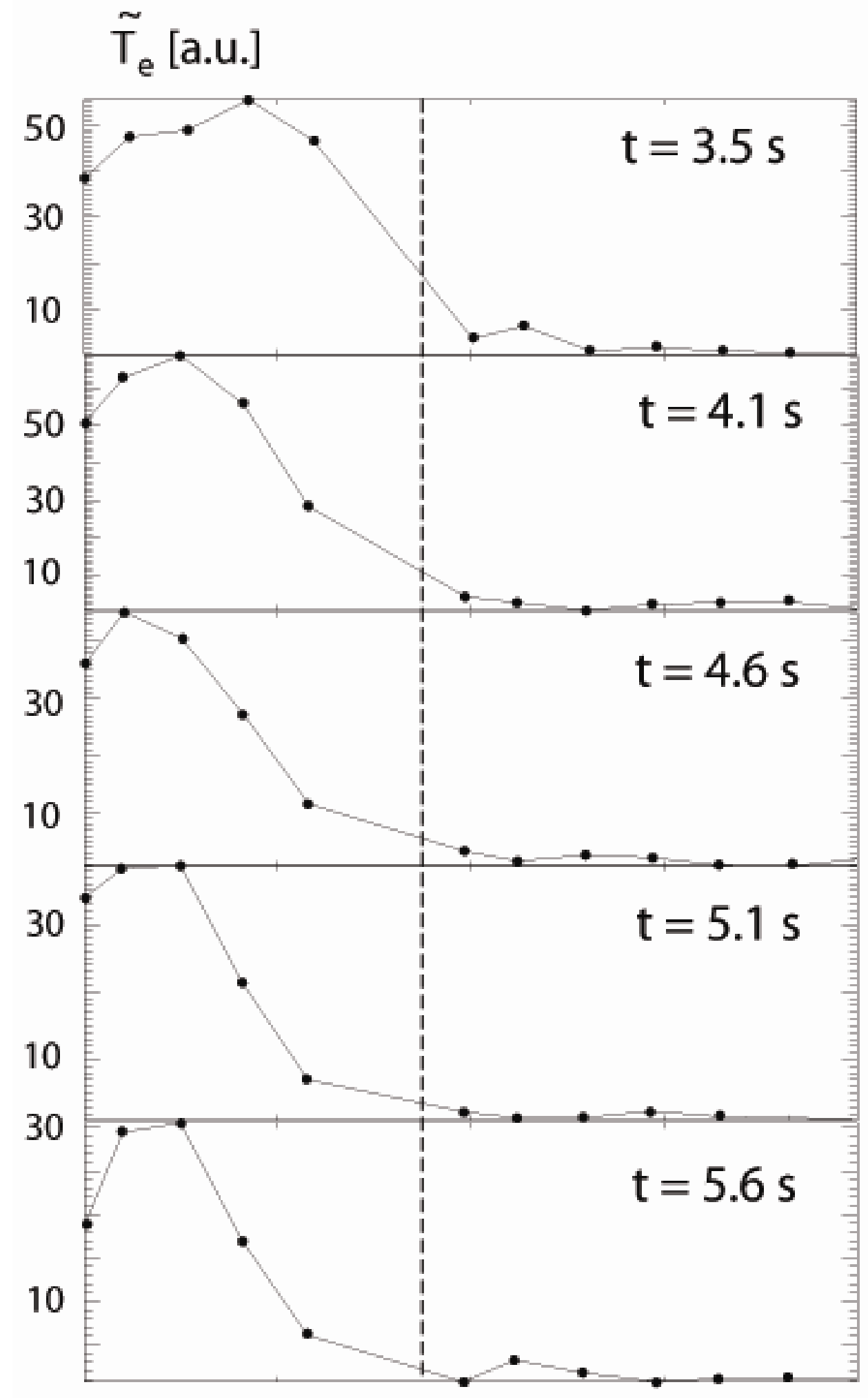

\section{$\begin{array}{llllll}0.04 & 0.15 & 0.27 & 0.38 & 0.49 & r_{\text {pol }}\end{array}$}

Fig. 6: Amplitude of the temperature perturbations (ECE) caused by small fishbones in a discharge with one beam only(\#18091). Discharge parameters: $800 \mathrm{kA}, B_{t}=2.5 \mathrm{~T}, \delta=0.2, P_{N B I}=2.5 M W, n_{e}=3.5$ 
$10^{19} \mathrm{~m}^{-3}$. At 3.5s the on-axis beam was replaced by an off-axis beam, the electron temperature was kept constant by additional central ICRH.

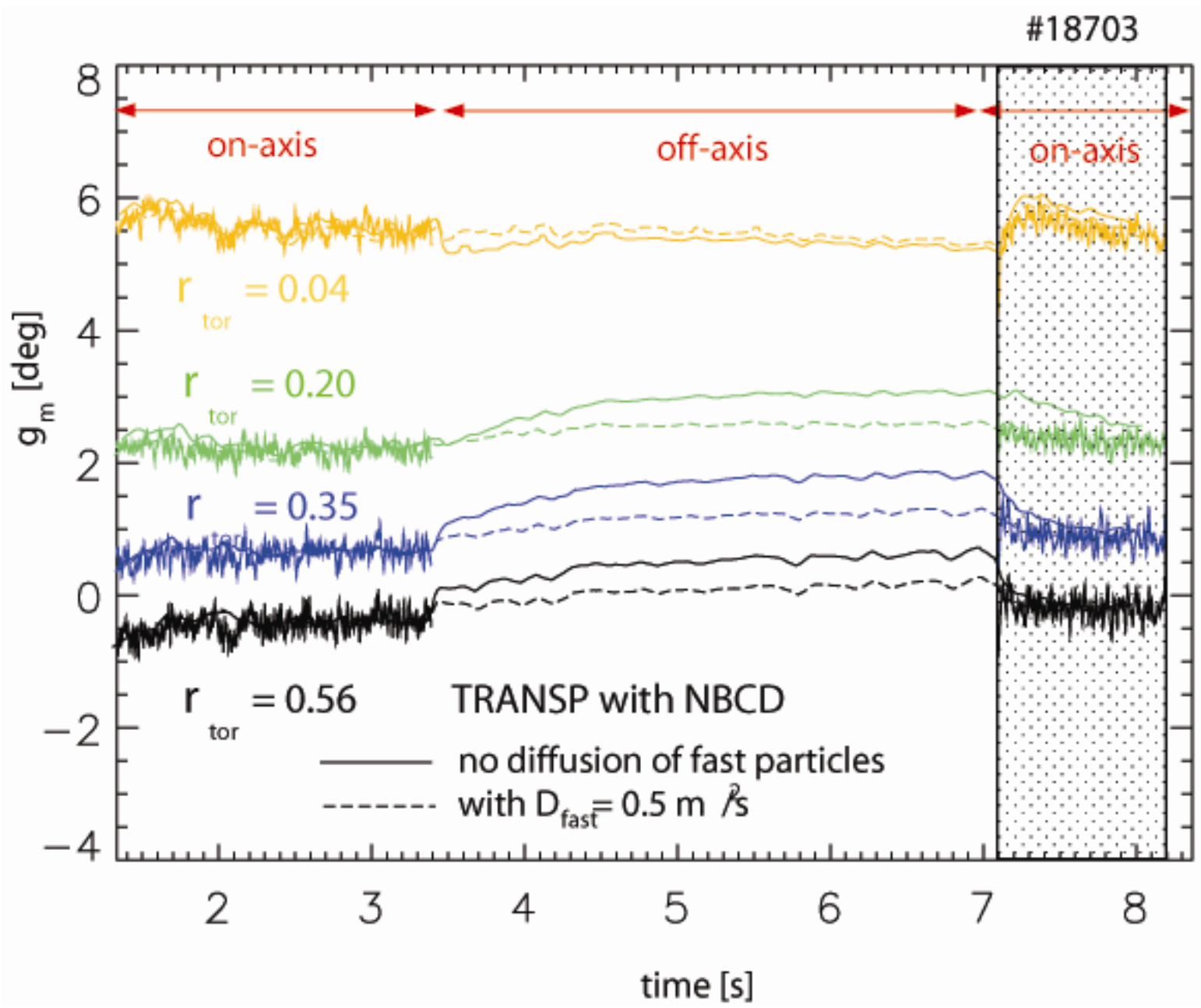

Fig.7: Measured time traces of the MSE diagnostic at different radii compared to TRANSP predictions including NBI current drive, without (solid) and with (dashed) artificially introduced fast particle diffusion. Discharge parameters: $I_{p}=600 \mathrm{kA}, B_{t}=2.5 \mathrm{~T}, \delta=0.2, P_{N B I}=5 \mathrm{MW}, q_{95}=6.2$. Note that the current profile relaxation right after the off-axis NBI phase (highlighted) agrees with TRANSP simulations only if fast particle diffusion is included. (The relative error of the MSE angles is about 0.2 degrees.) 


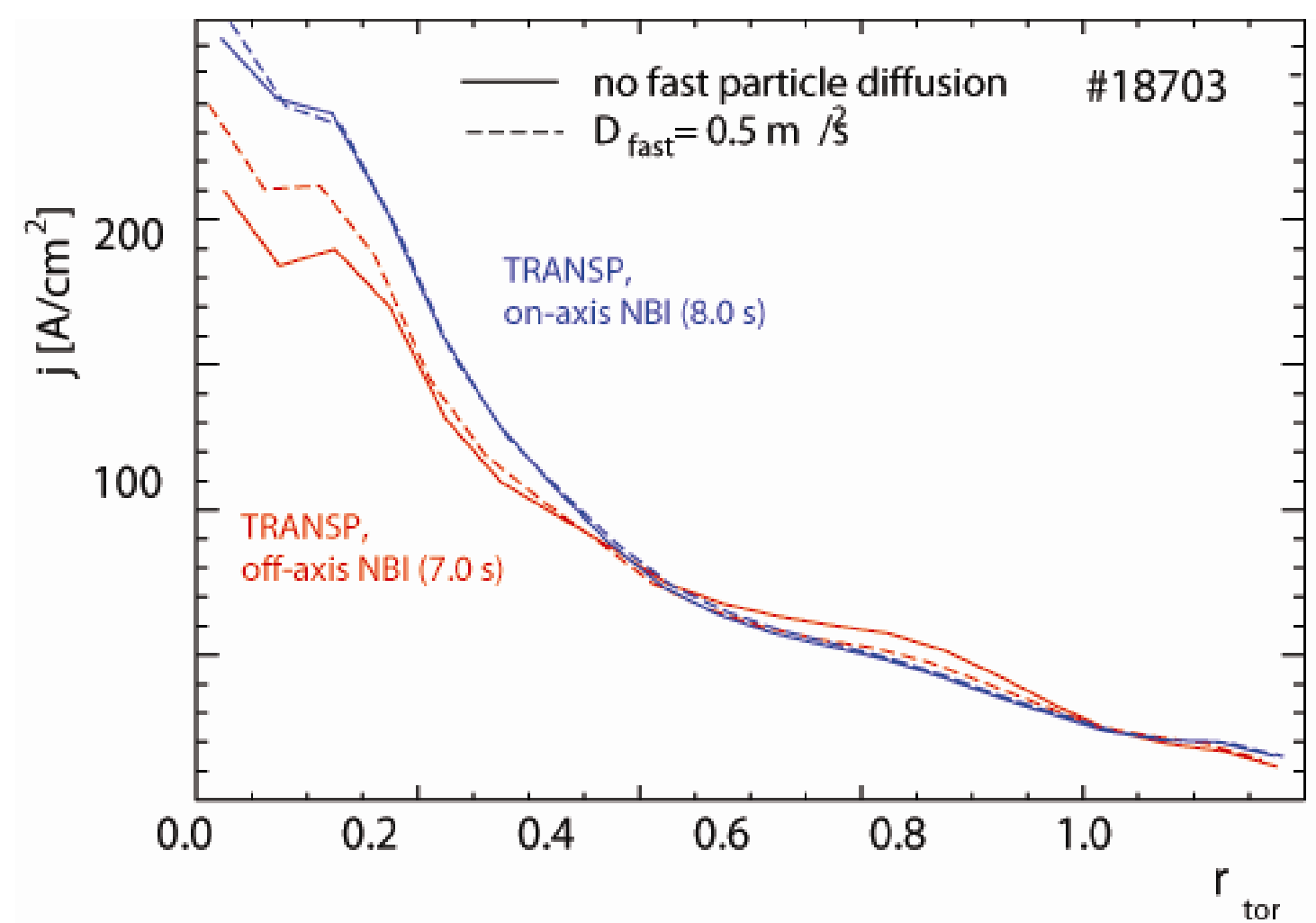

Fig. 8: TRANSP current density profiles at the end of the off-axis and the on-axis phases with (dashed) and without (solid) fast particle diffusion for the discharge of Fig. 6.

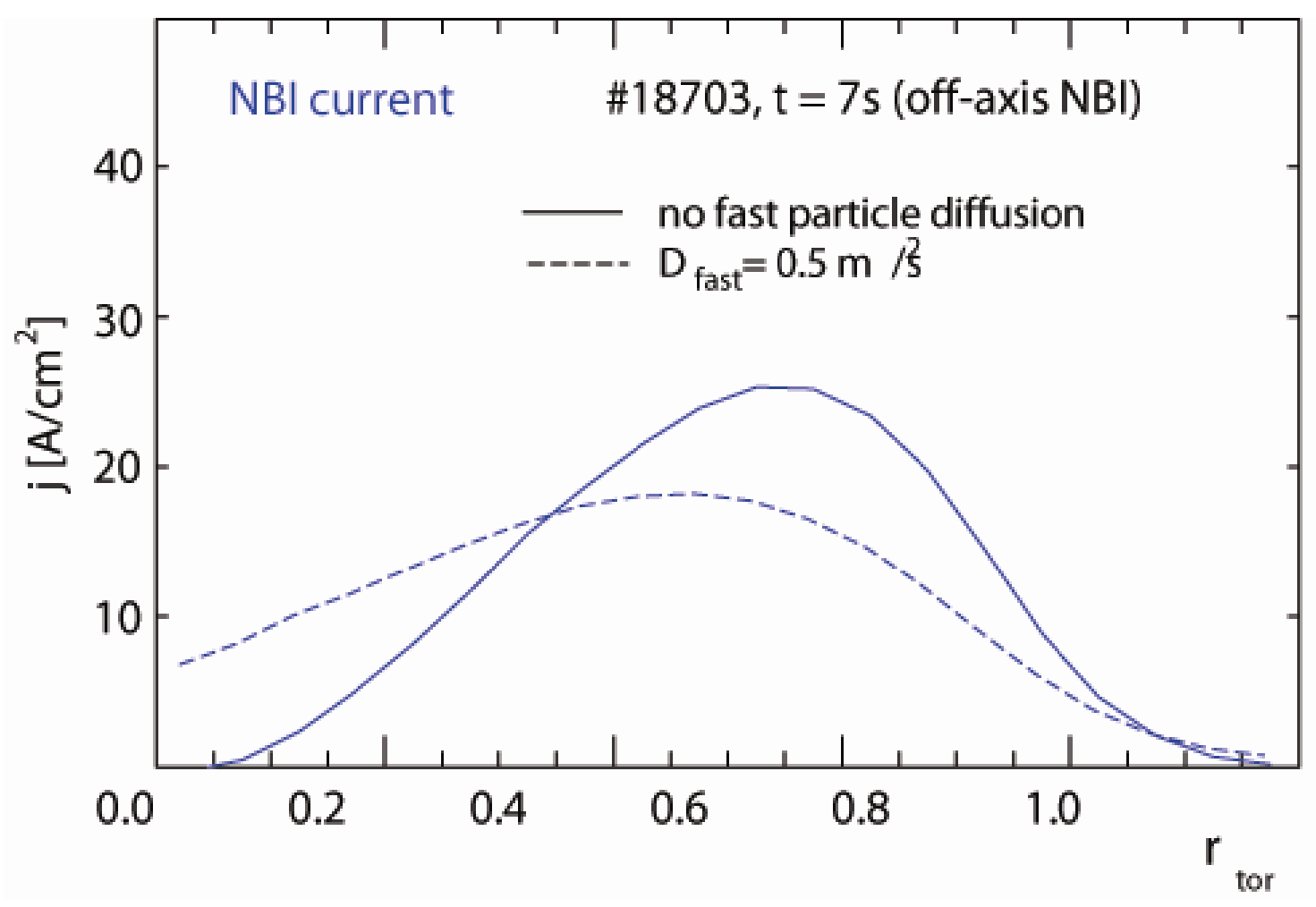

Fig. 9: TRANSP results for NBI current density profiles for off-axis NBI, without (solid) and with (dashed) artificially introduced fast particle diffusion for the discharge of Figs. 6 and 7. 


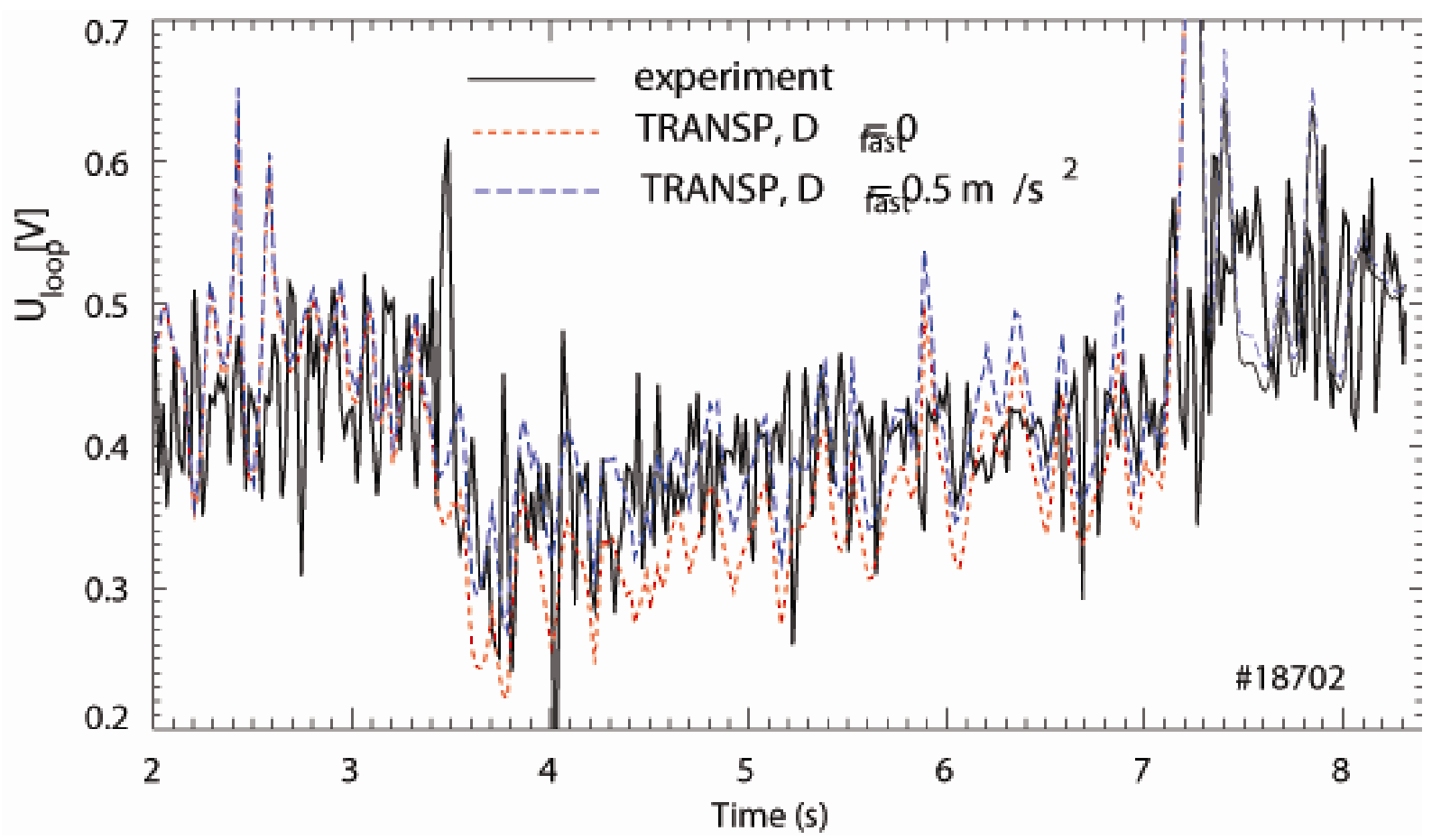

Fig.10: TRANSP prediction for the loop voltage at the plasma edge without (solid) and with (dashed) fast particle redistribution compared with measured loop voltage. Discharge parameters: $I_{p}=800 \mathrm{kA}$, $B_{t}=2.5 T, \delta=0.2, P_{N B I}=5 \mathrm{MW}, q_{95}=4.5$. For the TRANSP simulations a constant $Z_{\text {eff }}$ profile with $Z_{\text {eff }}=1.7$ was assumed. For the temperature measurements, Thomson measurements have been used.
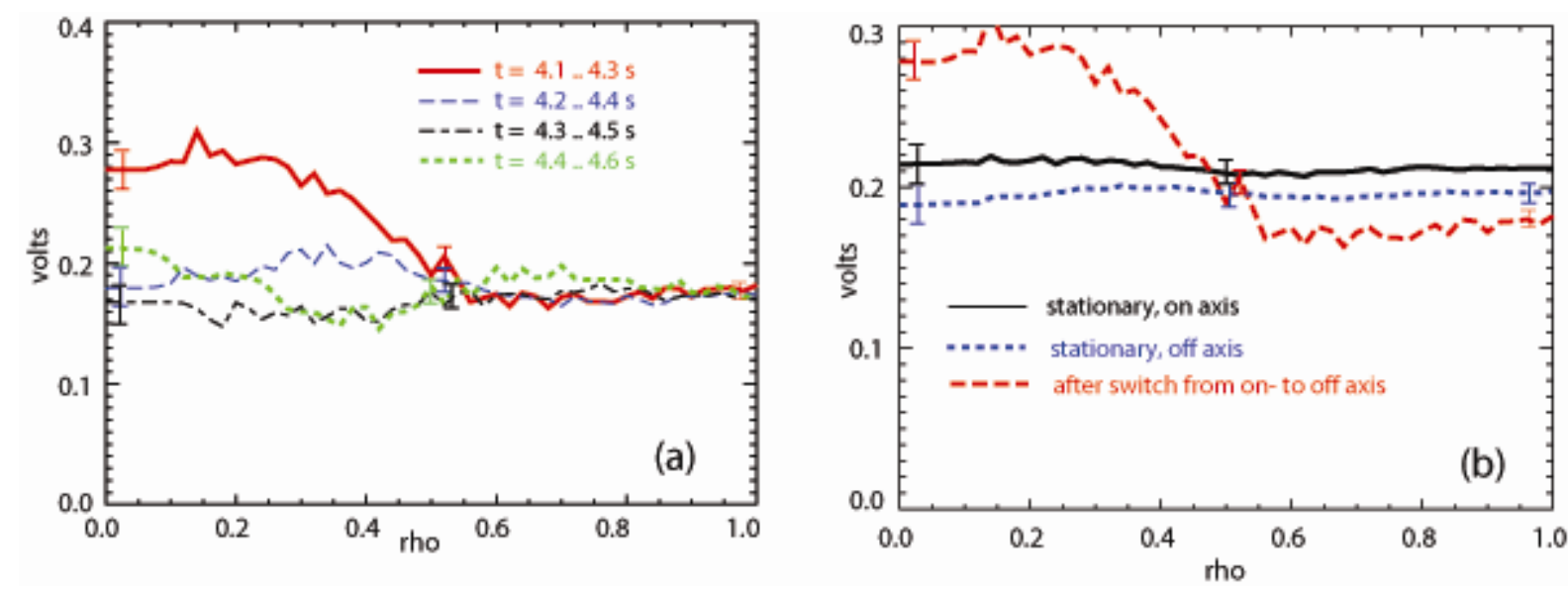

Fig. 11:(a)Time sequence of radial loop voltage profiles for the ASDEX Upgrade discharge \#19174 (averaged over $200 \mathrm{~ms}$ ) after switching from on- to off-axis beams at $4.1 \mathrm{~s}$. (b)t: Stationary loop voltage profile for the on-axis beam phase, the beginning of the off-axis phase and the stationary loop voltage profile for the off-axis beam phase. The difference in the stationary loop voltages demonstrates the larger current drive capability by the off axis beam. The total heating power is $5 \mathrm{MW}$ in a low triangularity discharge, the off-axis beams are reduced in voltage to restrict the fast ion velocity below $v_{A} / 3$. 


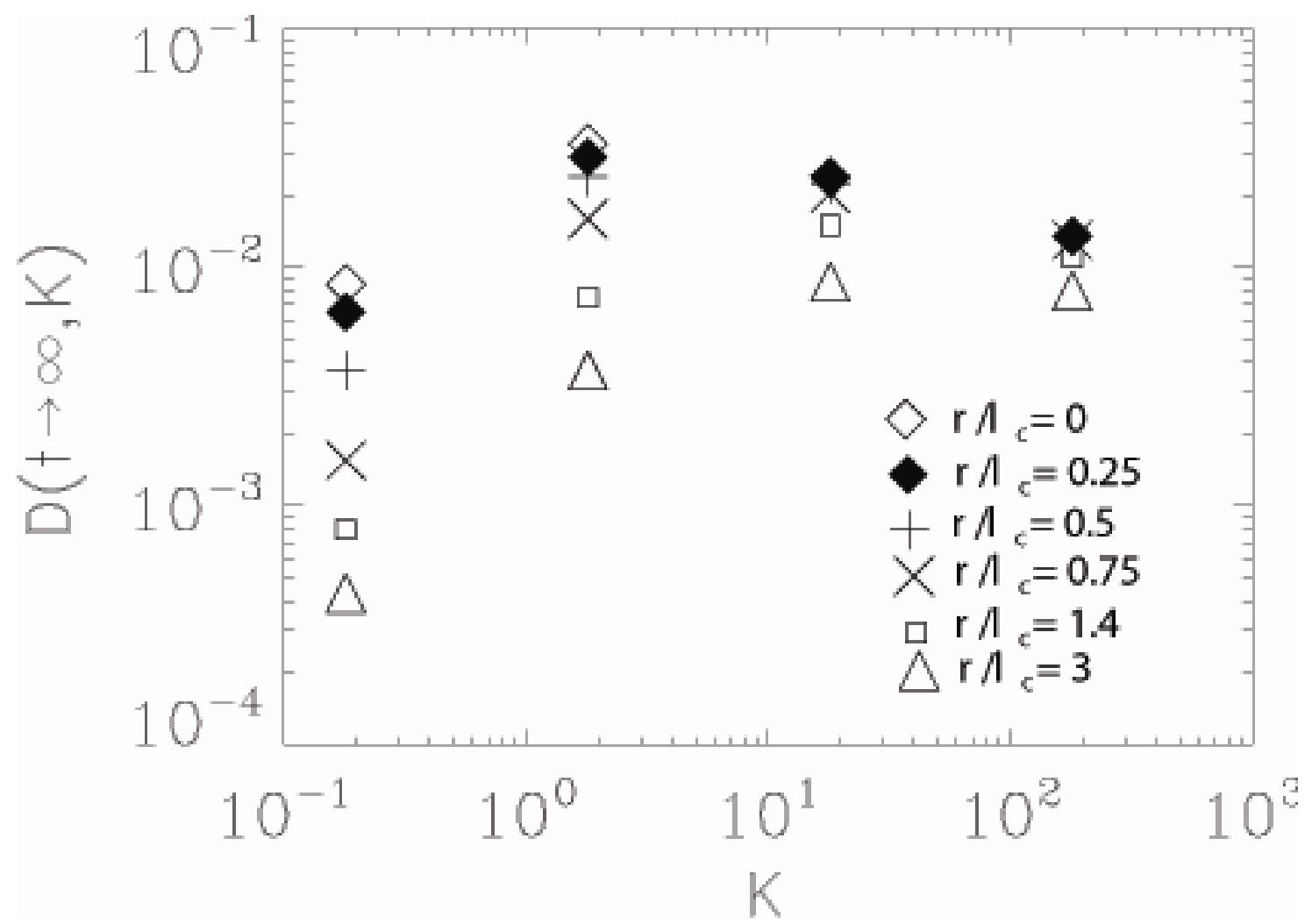

Fig. 12 Long-time limit of the diffusivity $D$ as a function of the Kubo number $K$ for different gyroradii $\rho$, normalized to the correlation length of the potential $\lambda_{c}$

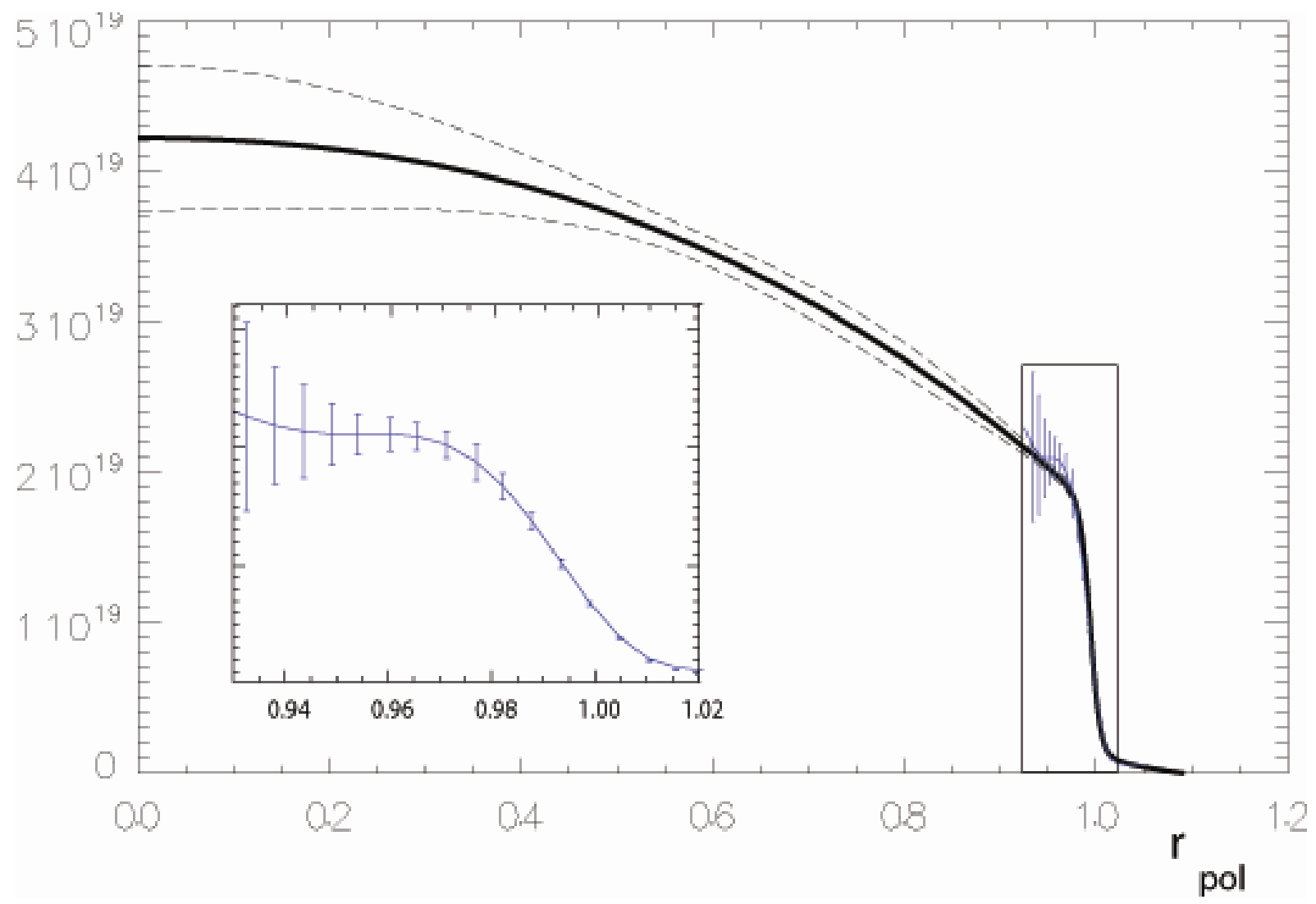


Fig: 13: Density profile for \#21007 ( $t=4.23 s)$ resulting from Li beam spectroscopy (edge) and DCN interferometry (core). The profile at the plasma edge is very well resolved.

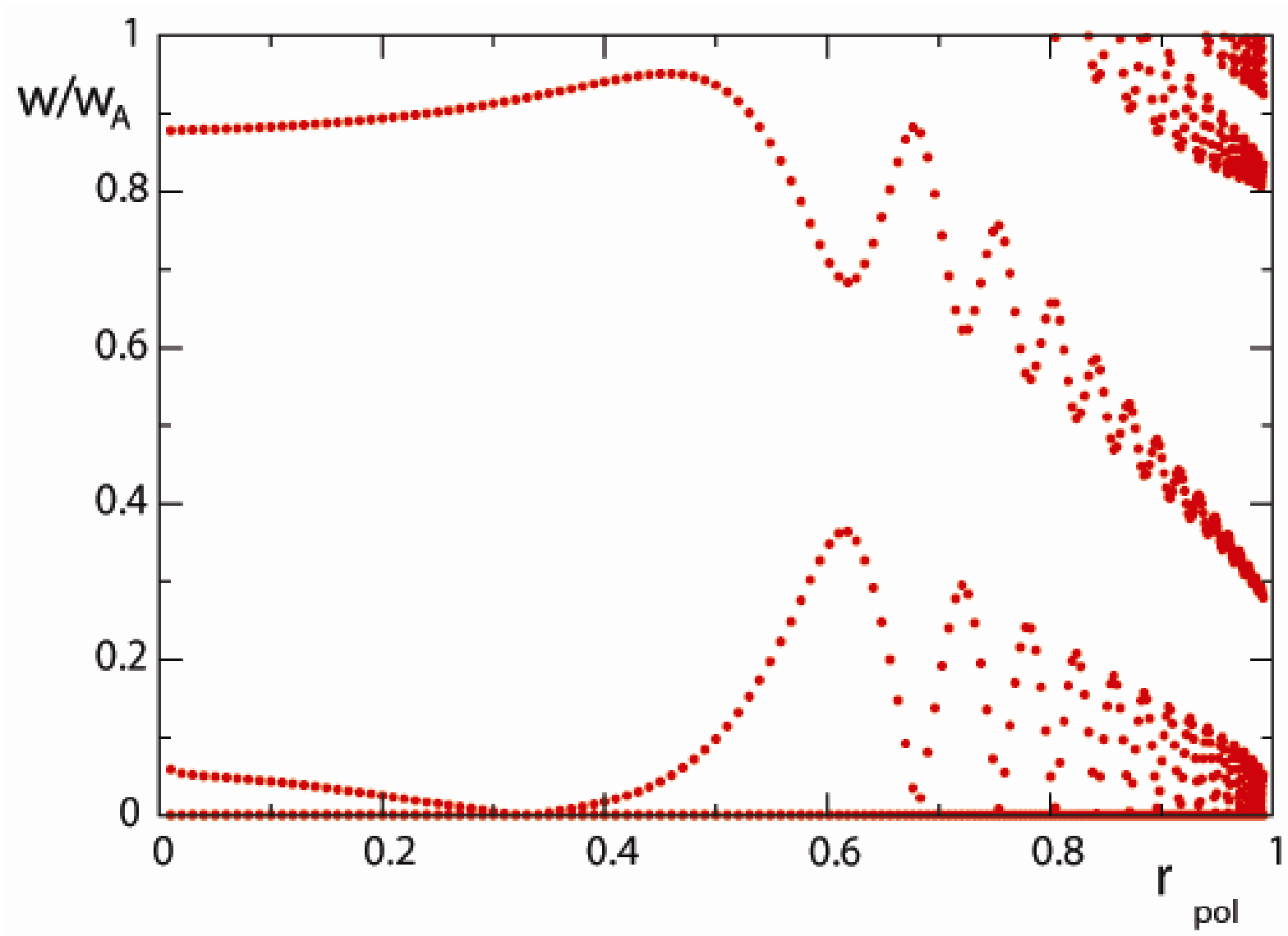

Fig. 14 n=4 Alfvén spectrum for \#21007, using the density profile of Fig. 13. Note the closed gap at the plasma edge.
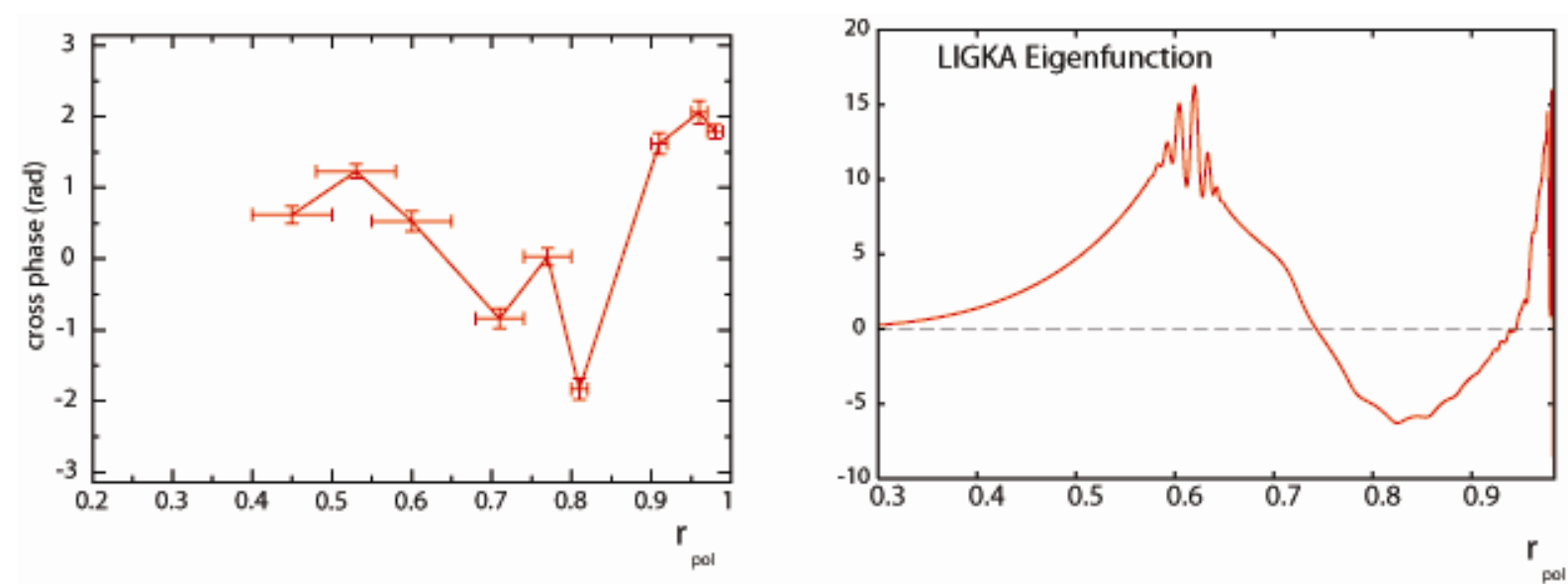

Fig. 15 Left: Radial profile of the cross phase between reflectometry and magnetic measurements for an $n=4$ TAE mode, taken from Ref. [37]. Right: LIGKA-density perturbation at the outer midplane according to Eq. 1 in [39] for the least damped $n=4$ TAE mode. 


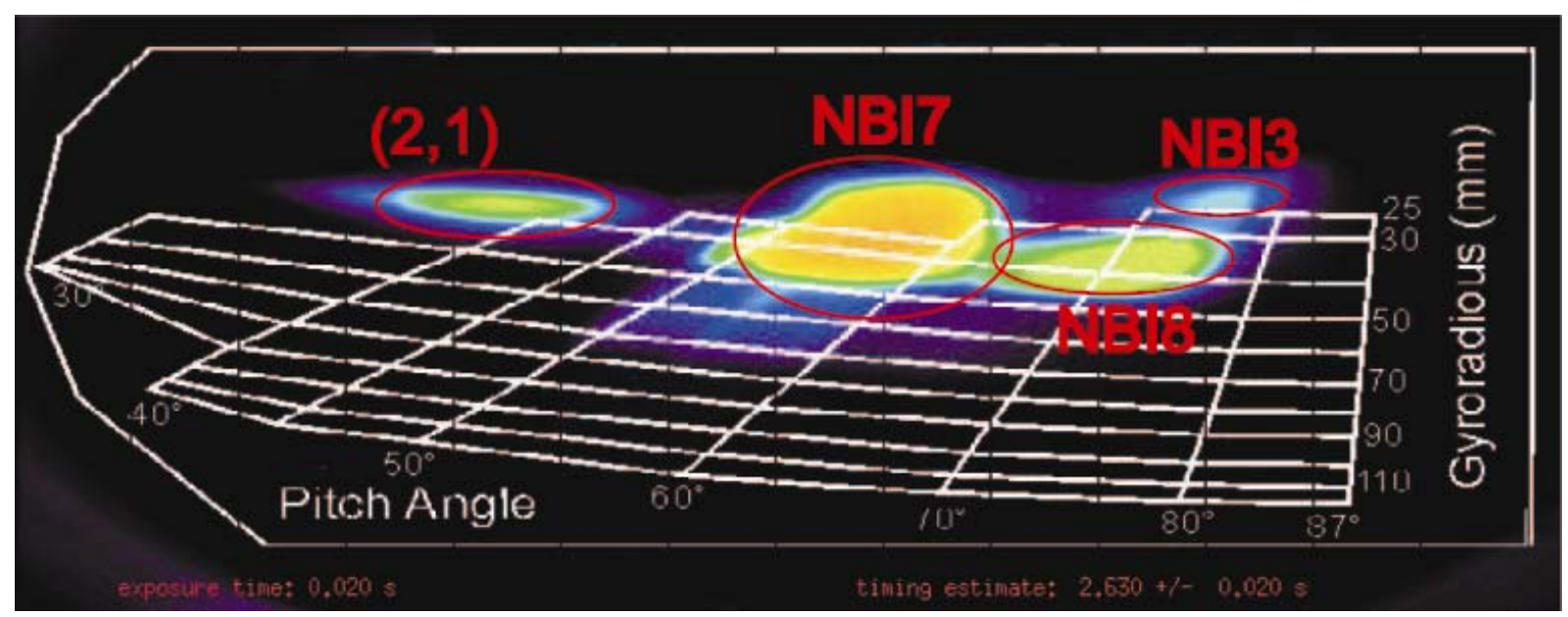

Fig. 16: CCD view of the light pattern produced by ion impacts on the scintillator plate for ASDEX Upgrade \#21168. The three most prominent signals correspond (from the left) to high field side deposition of the more radial $93 \mathrm{keV}$ source (source \#8), low field side deposited particles of the more tangential source (source \#7) and low field side deposition of \#8.
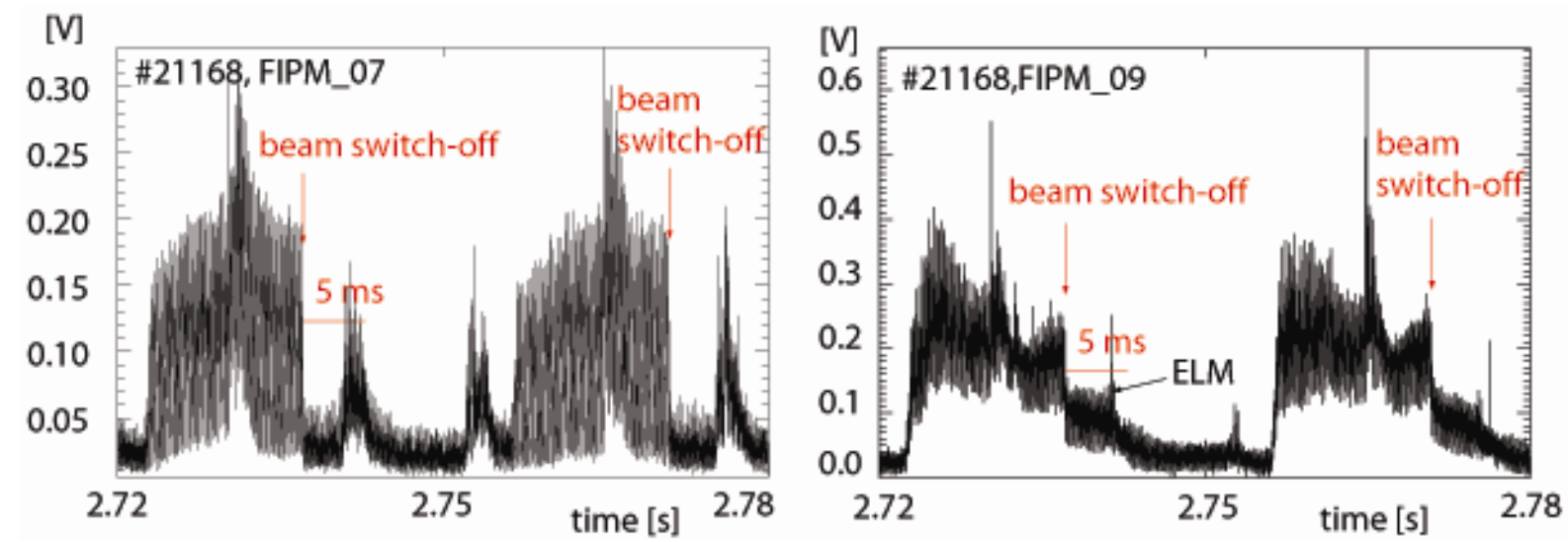

Fig. 17: Response of the fast ion losses to switch-on and-off of the NBI source \#8. Left for particles deposited on the high field side, right for particles deposited on the low field side. 


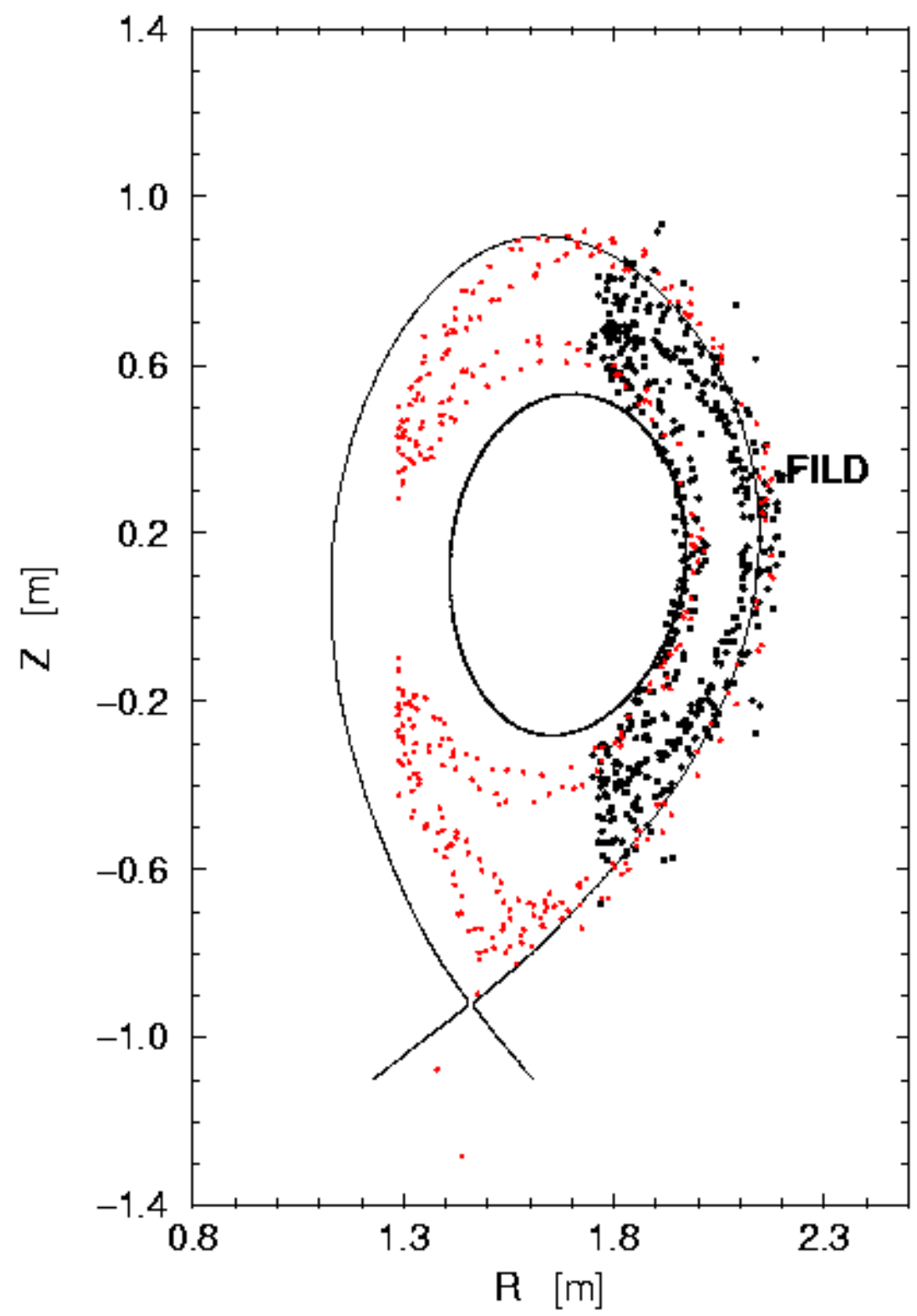

Fig. 18: Drift orbits for NBI particles (93 keV) deposited on the low field side for \#21168as resulting from GOURDON simulations. The solid line corresponds to the $q=2$ surface. The size of the $(2,1)$ magnetic islands is about $12 \mathrm{~cm}$. The position of the fast ion loss detector (FILD) is included. 

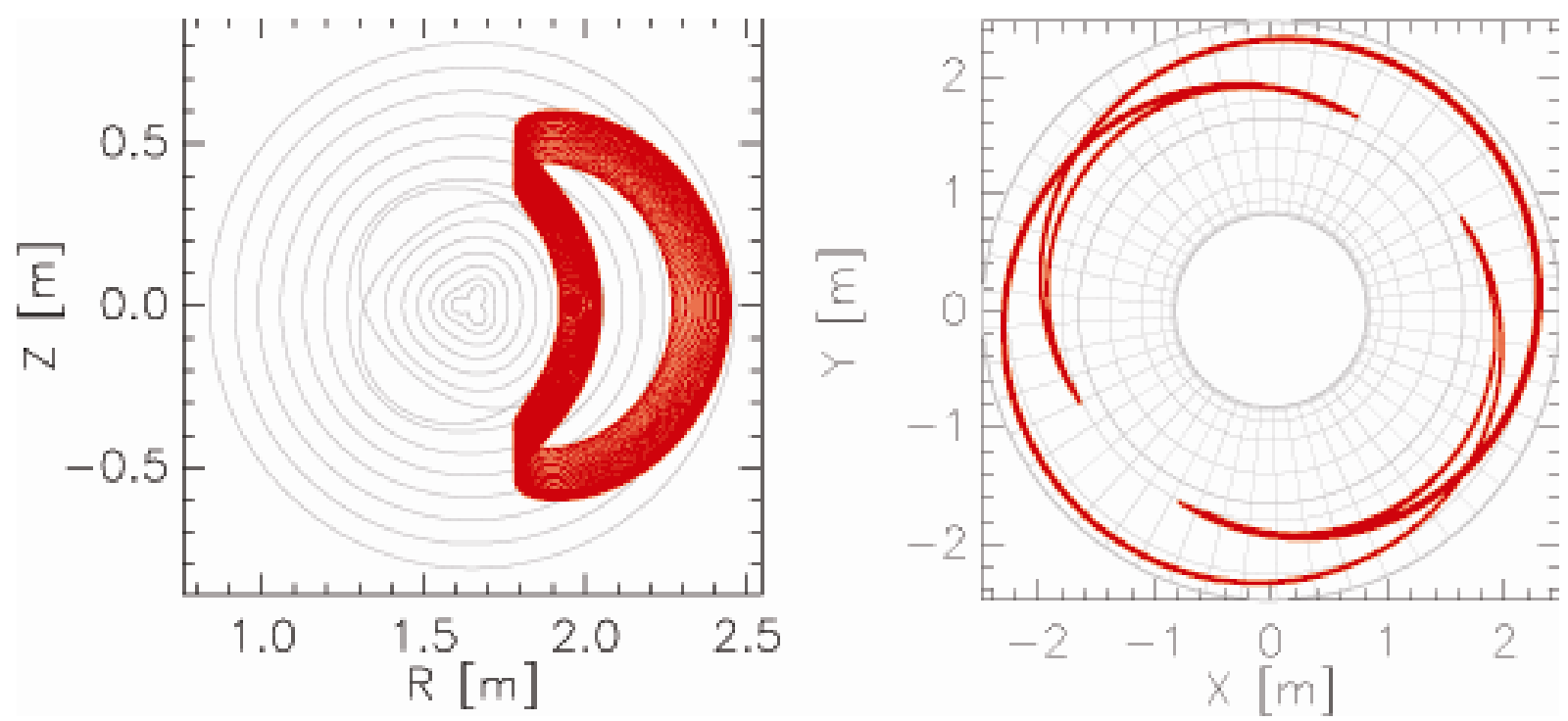

Fig. 19: Radial drift of an ICRH produced particle (energy: $600 \mathrm{keV}$ ) according to HAGIS calculations due to an $n=2$ magnetic island (left). The particle orbit shows an $n=2$ symmetry, allowing for a constant phase relation between particle and magnetic island (right). 
